\title{
Arritmias Cardíacas em Pacientes com COVID-19
}

\author{
Cardiac Arrhythmias in Patients with COVID-19
}

Mauricio Pimentel, ${ }^{10}$ Ana Paula Arbo Magalhães, ${ }^{1}$ Camila Valvassori Novak, ${ }^{1}{ }^{\circledR 0}$ Bruna Miers May, ${ }^{2}{ }^{\circledR 0}$ Luiz Gustavo Bravosi da Rosa, ${ }^{1}$ Leandro loschpe Zimerman ${ }^{10}$

Hospital de Clinicas de Porto Alegre, ${ }^{1}$ Porto Alegre, RS - Brasil

Programa de Pós-Graduação em Ciências da Saúde: Cardiologia e Ciências Cardiovasculares - Universidade Federal do Rio Grande do Sul, ${ }^{2}$

Porto Alegre, RS - Brasil

\section{Resumo}

Fundamento: A doença pelo novo coronavírus (COVID-19) está associada a manifestações clínicas cardiovasculares, incluindo a ocorrência de arritmias cardíacas.

Objetivos: Avaliar a incidência de arritmias cardíacas (taquiarritmia atrial, bradiarritmia e taquicardia ventricular sustentada) e de parada cardiorrespiratória (PCR) em uma coorte de pacientes internados com COVID-19 em hospital universitário terciário.

Métodos: Estudo de coorte retrospectivo realizado por meio de revisão dos registros de prontuário médico. Para comparação entre os grupos, foi considerado como estatisticamente significativo valor de $\mathbf{P}<0,05$.

Resultados: Foram incluídos 241 pacientes consecutivos com diagnóstico de COVID-19 (idade média, 57,8 \pm 15,0 anos; 51,5\% homens; $80,5 \%$ de raça branca) e 35,3\% com necessidade de ventilação mecânica invasiva (VM). A mortalidade geral foi de $26,6 \%$, sendo de $58,8 \%$ entre aqueles em VM. Arritmias cardíacas ocorreram em $8,7 \%$ dos pacientes, sendo a mais comum taquiarritmia atrial $(\mathbf{7 6}, 2 \%)$. Pacientes com arritmias apresentaram maior mortalidade, 52,4\% versus $\mathbf{2 4 , 1} \%(p=0,005)$. Em análise multivariada, apenas a presença de insuficiência cardíaca foi associada a maior risco de arritmias (hazard ratio, 11,9; IC 95\%: 3,6-39,5; p<0,001). Durante a internação, 3,3\% dos pacientes foram atendidos em PCR, com predomínio de ritmos não chocáveis. Todos os atendidos em PCR evoluíram com óbito durante a internação.

Conclusão: A incidência de arritmias cardíacas em pacientes internados com COVID-19 em hospital terciário brasileiro foi de $\mathbf{8 , 7} \%$, sendo a mais comum taquiarritmias atrial. A presença de insuficiência cardíaca foi associada a maior risco de arritmias. Pacientes com COVID-19 atendidos em PCR apresentam elevada mortalidade.

Palavras-chave: COVID-19; Arritmias Cardíacas; Parada Cardiorrespiratória; Fibrilação Atrial.

\begin{abstract}
Background: The coronavirus disease 2019 (COVID-19) is associated with cardiovascular clinical manifestations, including cardiac arrhythmias. Objective: To assess the incidence of cardiac arrhythmias (atrial tachyarrhythmia, bradyarrhythmia, and sustained ventricular tachycardia) and cardiac arrest (CA) in a cohort of patients hospitalized with COVID-19 in a tertiary university-affiliated hospital.

Methods: Cohort study with retrospective analysis of electronic medical records. For comparison between groups, a value of $p<0.05$ was considered statistically significant

Results: We included 241 consecutive patients diagnosed with COVID-19 (mean age, $57.8 \pm 15.0$ years; $51.5 \%$ men; $80.5 \%$ white), 35.3\% of whom received invasive mechanical ventilation (MV). The overall mortality was $26.6 \%$, being $58.8 \%$ among those on MV. Cardiac arrhythmias were identified in $8.7 \%$ of the patients, the most common being atrial tachyarrhythmia (76.2\%). Patients with arrhythmias had higher mortality (52.4\% versus $24.1 \%, p=0.005)$. On multivariate analysis, only the presence of heart failure (HF) was associated with a higher risk of arrhythmias (hazard ratio, 11.9; 95\% Cl: 3.6-39.5; $p<0.001$ ). During hospitalization, 3.3\% of the patients experienced CA, with a predominance of nonshockable rhythms. All patients experiencing CA died during hospitalization.

Conclusions: The incidence of cardiac arrhythmias in patients admitted with COVID-19 to a Brazilian tertiary hospital was $8.7 \%$, and atrial tachyarrhythmia was the most common. Presence of HF was associated with an increased risk of arrhythmias. Patients with COVID-19 experiencing CA have high mortality.
\end{abstract}

Keywords: COVID-19; Cardiac Arrhythmias; Heart Arrest; Atrial Fibrillation.

Full texts in English - http://www.arquivosonline.com.br

Correspondência: Leandro loschpe Zimerman •

Hospital de Clinicas de Porto Alegre - Cardiologia - Ramiro Barcelos, 2350. CEP 90035-903, Porto Alegre, RS - Brasil

E-mail: lizimerman@gmail.com

Artigo recebido em 31/08/2020, revisado em 17/11/2020, aceito em 25/11/2020

DOI: https://doi.org/10.36660/abc.20200963 


\section{Introdução}

A doença causada pelo coronavírus da síndrome respiratória aguda grave 2 (SARS-CoV-2), denominada COVID-19, teve seus primeiros casos reportados na China e, em decorrência de sua rápida disseminação mundial, foi declarada pandemia pela Organização Mundial da Saúde em 11 de março de 2020. No Brasil, o número de casos confirmados de COVID-19 ultrapassou 3 milhões em agosto de 2020.

Nas descrições das séries de casos de COVID-19, foram reportadas complicações cardiovasculares, incluindo injúria miocárdica, arritmias, miocardite, insuficiência cardíaca e choque cardiogênico. ${ }^{2} \mathrm{O}$ dano ao sistema cardiovascular provavelmente tem causa multifatorial, incluindo lesão cardíaca direta pelo vírus, resposta inflamatória sistêmica exacerbada e fenômenos tromboembólicos. ${ }^{3} \mathrm{~A}$ ação do vírus por meio do receptor da enzima conversora de angotensina 2 e seu efeito de down regulation são fatores envolvidos na resposta inflamatória exacerbada. ${ }^{4}$ No caso das arritmias cardíacas, pode-se considerar também a possibilidade de efeitos próarrítmicos de drogas utilizadas para tratamento da COVID-19, hipóxia causada por envolvimento viral pulmonar, isquemia miocárdica, distúrbios hidroeletrolíticos, "strain" miocárdico e alterações de volume intravascular. ${ }^{5} \mathrm{~A}$ resposta inflamatória desbalanceada por células T helper dos tipos 1 e 2 constitui ainda outro mecanismo proposto para explicar inflamação e arritmogênese em pacientes com COVID-19. ${ }^{6}$ As primeiras séries de casos da China mostraram incidência de arritmias cardíacas de $17 \%$, podendo chegar a $44 \%$ em pacientes internados em unidade de terapia intensiva (UTI). ${ }^{7}$ Esses estudos, porém, não descreveram de modo detalhado o tipo e as características das arritmias apresentadas. Trabalhos mais recentes em centros norte-americanos apontam incidência geral de arritmias de $6 \%$ e de taquiarritmias atriais de $16 \% .^{8-10}$

Este trabalho tem como objetivo avaliar a incidência de parada cardiorrespiratória e de arritmias cardíacas em uma coorte de pacientes internados com COVID-19 em hospital universitário terciário brasileiro.

\section{Métodos}

Estudo de coorte incluindo pacientes consecutivos internados com diagnóstico de COVID-19 no Hospital de Clínicas de Porto Alegre, Rio Grande do Sul, a partir de 1 de março até 20 de julho de 2020. Foram analisados os primeiros 241 pacientes consecutivos que tiveram diagnóstico confirmado de infecção por SARS-CoV-2 através de RT-PCR de aspirado nasofaríngeo.

Todos os registros de prontuário médico eletrônico foram revisados para obtenção de informações demográficas e comorbidades e ainda de dados sobre desfecho da internação hospitalar (óbito ou alta hospitalar), necessidade de ventilação mecânica invasiva (VM) e ocorrência de parada cardiorrespiratória atendida e de arritmias cardíacas. Foram analisados os registros de evolução médica, de enfermagem e os traçados eletrocardiográficos quando disponíveis no sistema de prontuário eletrônico.

Para os casos de parada cardiorrespiratória, foi revisada informação sobre ritmo inicial, classificado em: fibrilação ventricular/taquicardia ventricular (FV/TV), assistolia, bradiarritmia e atividade elétrica sem pulso (AESP). A ocorrência de arritmias cardíacas foi definida pela presença de taquiarritmias atriais sustentadas (fibrilação atrial, flutter atrial), bradiarritmias e taquicardia ventricular sustentada. Não foram incluídas arritmias já presentes na admissão hospitalar, apenas casos incidentes durante a internação. O protocolo do estudo foi aprovado pelo Comitê de Ética em Pesquisa do Grupo de Pesquisa e Pós-Graduação do Hospital de Clínicas de Porto Alegre.

\section{Análise estatística}

As variáveis contínuas com distribuição normal foram descritas através de média e desvio-padrão. O tempo de internação não apresentou distribuição normal pelo teste Shapiro-Wilk, sendo apresentado na forma de mediana e intervalo interquartil e comparado com teste de MannWhitney. As características demográficas e clínicas foram comparadas entre pacientes com e sem arritmias cardíacas utilizando-se o teste $t$ de Student não pareado para variáveis contínuas e qui-quadrado para variáveis categóricas. A associação entre variáveis clínicas e a ocorrência de arritmias cardíacas foi avaliada com modelos de análise univariada e multivariada de Cox. Foi considerado como estatisticamente significativo um valor de $p$ bicaudal $<0,05$. Todas as análises foram realizadas utilizando o programa SPSS, versão 14.0 para Windows.

\section{Resultados}

Neste estudo de coorte foram incluídos 241 pacientes consecutivos internados com COVID-19 e idade média de $57,8 \pm 15,0$ anos, sendo $51,5 \%$ homens e $80,5 \%$ de raça branca. A mediana do tempo de internação foi de 9 (intervalo interquartil 5-17) dias, sendo que 35,3\% dos pacientes necessitaram de VM. O tempo de internação foi maior naqueles que apresentaram arritmias cardíacas. A mortalidade geral foi de $26,6 \%$, sendo $58,8 \%$ entre os que necessitaram de VM e $9 \%$ entre os que não necessitaram de VM $(p=0,001)$.

A ocorrência de arritmias cardíacas, definida pela presença de taquiarritmias atriais sustentadas, bradiarritmias e taquicardia ventricular sustentada, foi observada em 21 pacientes $(8,7 \%)$. A Tabela 1 mostra as características demográficas e clínicas dos pacientes com e sem arritmias. Entre aqueles com arritmias, 16 (76,2\%) apresentaram taquiarritmias atriais sustentadas, $3(9,5 \%)$ taquicardia ventricular sustentada e $2(9,5 \%)$ bradiarritmias. Pacientes com arritmias apresentaram maior mortalidade, $52,4 \%$ versus $24,1 \%(p=0,005)$. A ocorrência de arritmias cardíacas foi mais frequente em homens, pacientes em VM e com história de insuficiência cardíaca. A Tabela 2 mostra os resultados da análise univariada e multivariada de Cox para ocorrência de arritmias cardíacas. Nesse modelo, apenas a presença de insuficiência cardíaca foi associada significativamente a maior risco de arritmias cardíacas (hazard ratio, 11,9; IC 95\% 3,6-39,5; $p<0,001)$. Em modelo ajustado para a presença de insuficiência cardíaca, a ocorrência de arritmias cardíacas foi associada a maior risco de mortalidade total (hazard ratio, 3,4; IC 95\% 1,8-6,7; $p<0,05$ ). 


\begin{tabular}{|c|c|c|c|c|}
\hline & $\begin{array}{l}\text { Todos os pacientes } \\
\qquad(n=241)\end{array}$ & $\begin{array}{l}\text { Com arritmia } \\
\qquad(n=21)\end{array}$ & $\begin{array}{l}\text { Sem arritmia } \\
\quad(n=220)\end{array}$ & Valor $p$ \\
\hline Idade, anos & $57,8 \pm 15,0$ & $62,6 \pm 13,4$ & $57,3 \pm 15,0$ & 0,11 \\
\hline Homens & $124(51,5)$ & $15(72,4)$ & $109(49,5)$ & 0,05 \\
\hline $\mathrm{IMC}, \mathrm{kg} / \mathrm{m}^{2}$ & $30,4 \pm 6,3$ & $29,3 \pm 5,0$ & $30,5 \pm 6,4$ & 0,43 \\
\hline Raça branca & $194(80,5)$ & $17(81)$ & $177(80,5)$ & 0,24 \\
\hline Ventilação mecânica & $85(35,3)$ & $14(66,7)$ & $71(32,2)$ & 0,002 \\
\hline Internação, dias & $9(5-17)$ & $25(12-43)$ & $9(5-16)$ & 0,001 \\
\hline Óbito & $64(26,6)$ & $11(52,4)$ & $53(24,1)$ & 0,005 \\
\hline \multicolumn{5}{|l|}{ Comorbidades } \\
\hline HAS & $123(51)$ & $14(66)$ & $109(49,5)$ & 0,13 \\
\hline DM & $64(26,6)$ & $7(33,3)$ & $57(25,9)$ & 0,46 \\
\hline IC & $15(6,2)$ & $5(23,8)$ & $10(4,5)$ & 0,001 \\
\hline Doença pulmonar & $52(21,6)$ & $7(33,3)$ & $45(20,5)$ & 0,17 \\
\hline Doença renal crônica & $29(12)$ & - & $29(13,2)$ & 0,07 \\
\hline \multicolumn{5}{|l|}{ Medicações } \\
\hline Hidroxicloroquina & $43(17,8)$ & $3(14,3)$ & $40(18,2)$ & 0,65 \\
\hline Anticoagulantes & $39(16,2)$ & $2(9,5)$ & $37(16,8)$ & 0,24 \\
\hline
\end{tabular}

Dados expressos como média \pm desvio-padrão ou números absolutos (percentual). 0 tempo de internação foi expresso pela mediana e intervalo interquartil. IMC: índice de massa corporal; HAS: hipertensão arterial sistêmica; DM: diabete melito; IC: insuficiência cardíaca.

Tabela 2 - Análise univariada e multivariada para o desfecho arritmias cardíacas

\begin{tabular}{lccccccc}
\hline & \multicolumn{3}{c}{ Análise univariada } & \multicolumn{3}{c}{ Análise multivariada } \\
\cline { 2 - 8 } & HR & IC $95 \%$ & $\mathbf{p}$ & HR & IC 95\% & p \\
\hline Homens & 2,04 & $0,79-5,32$ & 0,14 & 1,65 & $0,62-4,40$ & 0,31 \\
\hline Ventilação mecânica & 2,13 & $0,75-6,04$ & 0,15 & 2,57 & $0,88-7,49$ & 0,08 \\
\hline Insuficiência cardíaca & 11,10 & $3,48-35,3$ & 0,01 & 11,91 & $3,59-39,46$ & 0,01 \\
\hline
\end{tabular}

HR: hazard ratio; IC: intervalo de confiança.

Durante o período de internação, 8 pacientes $(3,3 \%)$, todos admitidos em UTI, foram atendidos em parada cardiorrespiratória e suas características clínicas são apresentadas na Tabela 3. A Figura 1 mostra a distribuição dos ritmos de parada cardiorrespiratória, sendo FV/TV em 2 pacientes (25\%), AESP em 3 (37,5\%) e assistolia em 3 (37,5\%). Todos os pacientes atendidos em parada cardiorrespiratória evoluíram para óbito durante a internação.

\section{Discussão}

Neste estudo de coorte que incluiu pacientes consecutivos internados com COVID-19 em hospital de referência, a mortalidade geral foi de $26,6 \%$, a incidência de arritmias, de $8,7 \%$ e a de parada cardiorrespiratória, de 3,3\%. A taquiarritmia atrial foi mais comum, correspondendo a $76,2 \%$ das arritmias. A presença de insuficiência cardíaca foi a única variável associada a maior risco de arritmias cardíacas em análise multivariada. O tempo de internação de pacientes com arritmias foi maior do que o daqueles sem; isso pode se dever à necessidade de tratamento da própria arritmia ou representar a presença de maior complexidade e gravidade nos casos que desenvolvem arritmias.

As séries iniciais mostraram incidência de arritmias cardíacas em pacientes com COVID-19 variando de 7\% a $17 \%$, porém não há descrição específica sobre o seu tipo. ${ }^{7,10}$ Na coorte do estado de Nova York, a incidência de arritmias foi associada a diferentes combinações de drogas utilizadas para tratamento da COVID-19, variando de $10 \%$ a 20\%, porém sem definição dos tipos de arritmia avaliados. ${ }^{11}$ Em nosso estudo, não encontramos associação do uso de hidroxicloroquina com maior risco de arritmias. A incidência específica de cada tipo de arritmia foi descrita apenas recentemente. Dados de registro internacional que incluiu 1197 profissionais de eletrofisiologia mostram que fibrilação atrial foi a arritmia mais frequentemente descrita em pacientes com COVID-19. ${ }^{12}$ Em estudo com 115 pacientes, a incidência de taquiarritmias atriais foi de $16,5 \%$, sendo de $27,5 \%$ entre os admitidos em UTI. ${ }^{8}$ No maior estudo específico sobre arritmias publicado 


\begin{tabular}{|c|c|c|c|c|}
\hline $\begin{array}{l}\text { Paciente } \\
\text { número }\end{array}$ & $\begin{array}{l}\text { Dia de internação } \\
\text { da PCR }\end{array}$ & Ritmo da PCR & Descrição clínica & Desfecho \\
\hline 1 & 1 & AESP & $\begin{array}{l}26 \text { anos, asma, obesidade e } \\
\text { esquizofrenia }\end{array}$ & $\begin{array}{l}\text { ROSC } 20 \text { minutos, encefalopatia anóxica, } \\
\text { instituídas medidas de conforto, óbito }\end{array}$ \\
\hline 2 & 26 & AESP & 54 anos, transplantada renal & $\begin{array}{l}\text { ROSC } 2 \text { minutos, evolução hospitalar com choque } \\
\text { refratário, óbito }\end{array}$ \\
\hline 3 & 25 & $\mathrm{FV} / \mathrm{TV}$ & $\begin{array}{l}58 \text { anos, miocardiopatia dilatada, } \\
\text { portador de CDI, SARA e VM }\end{array}$ & ROSC 20 minutos, choque refratário, óbito \\
\hline 5 & 10 & $\mathrm{FV} / \mathrm{TV}$ & 45 anos, miocardiopatia dilatada & ROSC 12 minutos, choque refratário, óbito \\
\hline 6 & 43 & Assistolia & 71 anos, cardiopatia isquêmica & Óbito \\
\hline 7 & 12 & Assistolia & 63 anos, HAS, DM & Óbito \\
\hline 8 & 01 & AESP & 76 anos, cardiopatia isquêmica & Óbito \\
\hline 9 & 25 & Assistolia & 41 anos, HAS, obesidade & ROSC 35 min, disfunção múltiplos órgãos, óbito \\
\hline
\end{tabular}

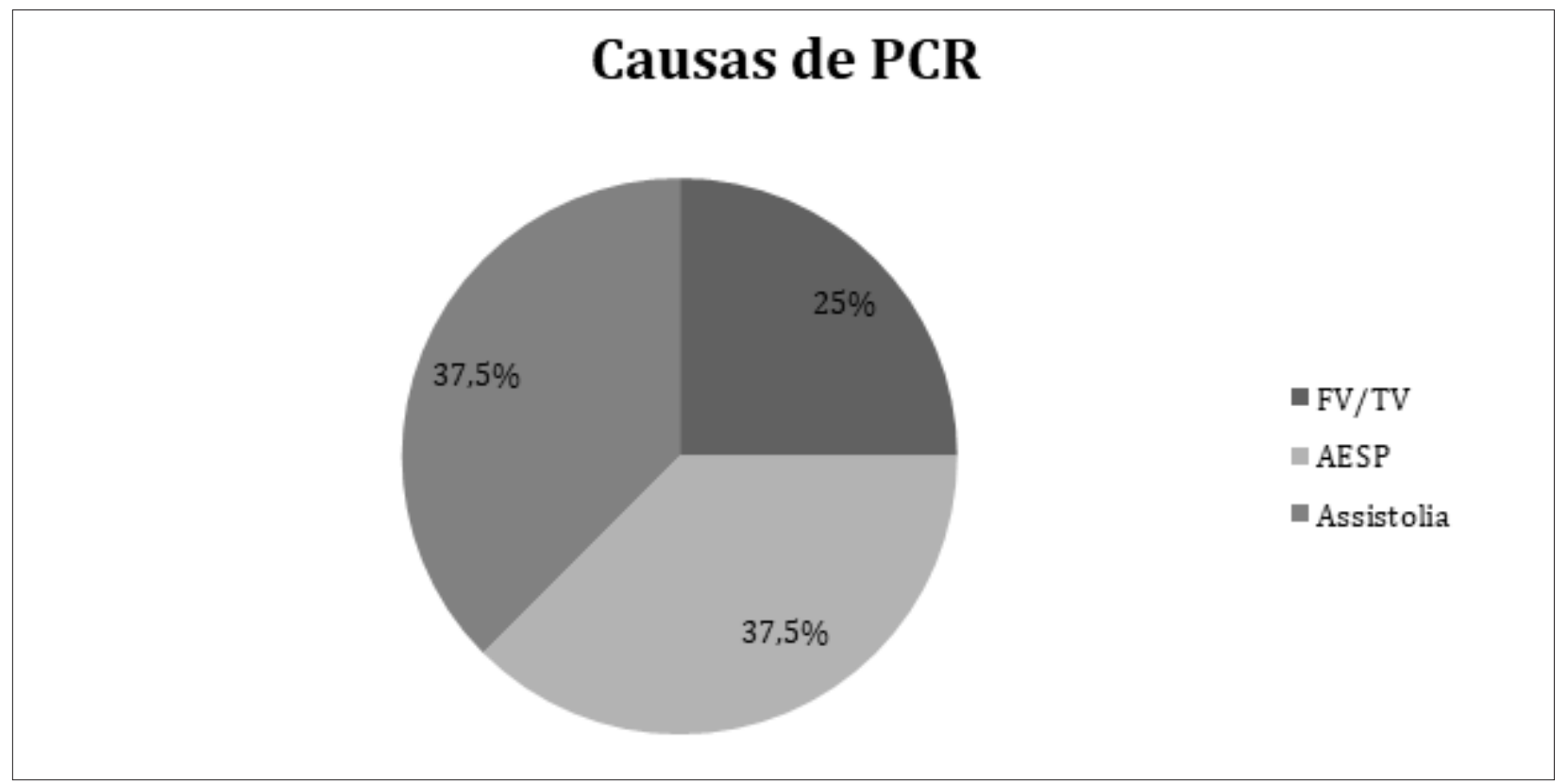

Figura 1 - Ritmos de parada cardiorrespiratória em pacientes com COVID-19. PCR: parada cardiorrespiratória; AESP: atividade elétrica sem pulso; FV/TV: fibrilação ventricular/taquicardia ventricular.

até o momento, foram avaliados 700 pacientes hospitalizados por 9 semanas. ${ }^{9}$ Durante o acompanhamento, 44 deles $(6,3 \%)$ apresentaram arritmias cardíacas, incluindo fibrilação atrial, bradiarritmias e taquicardia ventricular não sustentada, sendo fibrilação atrial a mais frequente (57\%). Presença de insuficiência cardíaca e internação em UTI foram associadas significativamente a maior risco de arritmias. Naquela coorte, $11 \%$ dos pacientes foram admitidos em UTI e a mortalidade geral foi de $4 \%$. Nossa incidência de arritmia de $8,7 \%$ pode ser considerada próxima à daquele estudo, assim como o predomínio de arritmias atriais e a associação da presença de insuficiência cardíaca com maior risco de arritmias. Por outro lado, em nosso estudo foram incluídos pacientes de maior gravidade (35\% com necessidade de VM) e a presença de taquicardia ventricular não sustentada não foi elencada como desfecho. A utilização de VM foi associada à tendência de maior risco de arritmias, porém não foi considerada estatisticamente significativa. A confirmação do achado de que pacientes com insuficiência cardíaca apresentam maior risco de arritmias pode orientar a necessidade de maior monitorização desses pacientes durante internação.

$\mathrm{Na}$ pesquisa internacional entre profissionais de eletrofisiologia, 4,8\% reportaram casos de FV/TV e 5,6\%, de AESP. ${ }^{12}$ Nas séries iniciais da China, não houve indicação 
específica sobre a ocorrência de parada cardiorrespiratória e seus ritmos. ${ }^{2,7} \mathrm{Em}$ um dos trabalhos, a incidência de FV/ TV foi de 5,9\%, sendo maior entre pacientes com troponina elevada. ${ }^{2} \mathrm{Na}$ coorte do estado de Nova York, a incidência de parada cardiorrespiratória descrita foi de $6 \%$ a $15 \%$, variando de acordo com diferentes combinações de drogas utilizadas no tratamento da COVID-19. ${ }^{11}$ Não foram descritos os ritmos de parada cardiorrespiratória. No estudo de Bhatla et al. já descrito acima, foram relatados 9 casos de parada cardiorrespiratória (1,3\%), sendo 6 casos de AESP, 2 de assistolia e 1 de torsades de pointes. ${ }^{9}$ Em nosso estudo, a incidência de parada cardiorrespiratória foi de 3,3\%, também com predomínio de ritmos não chocáveis. A redução da ocorrência de casos de FV/TV em relação a dos estudos iniciais pode hipoteticamente ser atribuída a mudanças no tratamento da COVID-19, com menor utilização de drogas que podem prolongar o intervalo QT, além da evolução da curva de aprendizado dos profissionais de saúde com a doença. O predomínio de ritmos não chocáveis também pode ser atribuído ao acometimento sistêmico e à intensa resposta inflamatória presente nos casos graves de COVID-19.

Nosso estudo apresenta limitações que devem ser consideradas. O número de pacientes incluídos é relativamente pequeno, refletindo a experiência inicial de atendimento. Os pacientes internados em enfermaria não estavam em monitorização cardíaca contínua, assim, episódios de arritmia assintomáticos podem não ter sido relatados. O diagnóstico de arritmias foi obtido a partir da revisão dos registros de prontuário, sendo que em alguns casos a arritmia descrita foi visualizada apenas em monitor e não foi feito o registro em eletrocardiograma de 12 derivações. Não foram obtidos dados laboratoriais de marcadores de lesão e/ou disfunção miocárdica, como troponina e BNP, dados sobre formas de ventilação não invasiva, momento e doses no uso de drogas vasoativas, distúrbios hidroeletrolíticos e histórico prévio de arritmias, todos potencialmente associados à ocorrência de arritmias durante a internação. Trata-se de estudo com coleta de dados retrospectiva e em centro único terciário. Dessa forma, seus resultados não podem ser generalizados para outros cenários clínicos.

\section{Referências}

1. Cornavírus Brasil [Internet]. Brasília: Ministério da Saúde; 2020 [cited 2021 Jun 23] Available from: https://covid.saude.gov.br/.

2. Guo T, Fan Y, Chen M, Wu X, Zhang L, He T, et al. Cardiovascular Implications of Fatal Outcomes of Patients with Coronavirus Disease 2019 (COVID-19). JAMA Cardiol. 2020;5(7):811-8. doi: 10.1001/jamacardio.2020.1017.

3. Costa IBSS, Bittar CS, Rizk SI, Araújo Filho AE, Santos KAQ, Machado TIV, et al. The Heart and COVID-19: What Cardiologists Need to Know. Arq Bras Cardiol. 2020;114(5):805-16. doi: 10.36660/abc.20200279.

4. Wong CK, Lam CW, Wu AK, Ip WK, Lee NL, Chan IH, et al. Plasma Inflammatory Cytokines and Chemokines in Severe Acute Respiratory Syndrome. Clin Exp Immunol. 2004;136(1):95-103. doi: 10.1111/j.13652249.2004.02415.x.

5. Dherange P, Lang J, Qian P, Oberfeld B, Sauer WH, Koplan B, et al. Arrhythmias and COVID-19: A Review. JACC Clin Electrophysiol. 2020;6(9):1193-204. doi: 10.1016/j.jacep.2020.08.002.

\section{Conclusões}

Neste estudo de coorte de pacientes com COVID-19 internados em hospital de referência brasileiro, a incidência de arritmias cardíacas foi de $8,7 \%$, sendo taquiarritmia atrial a mais comum. A presença de insuficiência cardíaca foi associada a maior risco de arritmias cardíacas. Pacientes com COVID-19 atendidos em parada cardiorrespiratória apresentam elevada mortalidade.

\section{Contribuição dos autores}

Concepção e desenho da pesquisa, Análise estatística, Redação do manuscrito e Revisão crítica do manuscrito quanto ao conteúdo intelectual importante: Pimentel M, Zimerman LI; Obtenção de dados: Pimentel M, Magalhães APA, Novak CV, May BM, Rosa LGB, Zimerman LI; Análise e interpretação dos dados: Pimentel M, Magalhães APA, Zimerman LI.

\section{Potencial conflito de interesse}

Não há conflito com o presente artigo

\section{Fontes de financiamento}

O presente estudo não teve fontes de financiamento externas.

\section{Vinculação acadêmica}

Não há vinculação deste estudo a programas de pósgraduação.

\section{Aprovação ética e consentimento informado}

Este estudo foi aprovado pelo Comitê de Ética do Hospital de Clínicas de Porto Alegre sob o número de protocolo 12744919500005327. Todos os procedimentos envolvidos nesse estudo estão de acordo com a Declaração de Helsinki de 1975, atualizada em 2013.
6. Cameron MJ, Bermejo-Martin JF, Danesh A, Muller MP, Kelvin DJ. Human Immunopathogenesis of Severe Acute Respiratory Syndrome (SARS). Virus Res. 2008;133(1):13-9. doi: 10.1016/j.virusres.2007.02.014.

7. Wang D, Hu B, Hu C, Zhu F, Liu X, Zhang J, et al. Clinical Characteristics of 138 Hospitalized Patients with 2019 Novel Coronavirus-Infected Pneumonia in Wuhan, China. JAMA. 2020;323(11):1061-9. doi: 10.1001/ jama.2020.1585.

8. Colon CM, Barrios JG, Chiles JW, McElwee SK, Russell DW, Maddox WR, et al. Atrial Arrhythmias in COVID-19 Patients. JACC Clin Electrophysiol. 2020;6(9):1189-90. doi: 10.1016/j.jacep.2020.05.015.

9. Bhatla A, Mayer MM, Adusumalli S, Hyman MC, Oh E, Tierney A, et al. COVID-19 and Cardiac Arrhythmias. Heart Rhythm. 2020;17(9):1439-44. doi: 10.1016/j.hrthm.2020.06.016.

10. Goyal P, Choi JJ, Pinheiro LC, Schenck EJ, Chen R, Jabri A, et al. Clinical Characteristics of Covid-19 in New York City. N Engl J Med. 2020;382(24):2372-4. doi: 10.1056/NEJMc2010419. 
11. Rosenberg ES, Dufort EM, Udo T, Wilberschied LA, Kumar J, Tesoriero J, et al. Association of Treatment With Hydroxychloroquine or Azithromycin With In-Hospital Mortality in Patients With COVID-19 in New York State. JAMA. 2020;323(24):2493-502. doi: 10.1001/jama.2020.8630.
12. Gopinathannair R, Merchant FM, Lakkireddy DR, Etheridge SP, Feigofsky S, Han JK, et al. COVID-19 and Cardiac Arrhythmias: A Global Perspective on Arrhythmia Characteristics and Management Strategies. J Interv Card Electrophysiol. 2020;59(2):329-36. doi: 10.1007/s10840-020-00789-9. 